\title{
REFLEXIONES SOBRE LA DIDÁCTICA DE LAS CIENCIAS SOCIALES: APORTES PARA LOS DOCENTES QUE ORIENTAN LA ENSEÑANZA DE LAS CIENCIAS SOCIALES EN LOS NIVELES EDUCATIVOS BÁSICO Y MEDIA
}

\begin{abstract}
Resumen
Los autores afirman que la finalidad de la enseñanza de las ciencias sociales no es formar científicos sociales ni expertos en una ciencia o disciplina. Lo que se enseña en ciencias sociales debe rebasar el conocimiento de las mismas y se encuentra ligado a la formación social de la escuela para que niños y adolescentes tengan herramientas necesarias para leer, interpretar, analizar criticamente y desarrollarse en la realidad en la que se hallan inmersos y trabajar para mejorarla. Así, pues, aquello que le interesaba a la didáctica clásica, que se remitía a los dispositivos metodológicos, debe ser replanteado, pues no sólo la condición postmoderna exige pensar en la información sino que el acontecimiento educativo implica otros elementos que no se habian tenido en cuenta, como el problema de las representaciones, de las transposiciones, que superan el problema de la conceptualización. Con todo, el interés de los autores de este texto ha sido plantearse cómo en el mundo educativo contemporáneo se puede repensar la didáctica de las ciencias sociales, con nuevos paradigmas y nuevas formas de enseñanza, adecuadas a la complejidad del mundo actual y fortaleciendo sus propósitos comprensivos y emancipadores del pensamiento crítico-social. En este orden de ideas, es fundamental que este texto sea un referente de reflexión para los docentes do ciencias sociales acerca de sus prácticas educativas y su formación disciplinar.
\end{abstract}

Palabras Clave: Didáctica, hermenéutica, pensamiento crítico, ciencias sociales.

* Candidata a Magister en Educación. Profesora - investigadora del Centro de Estudios de Didáctica y Pedagogia -CEDIP, Universidad de Ibagué. sandra.gutierrez@unibague.edu.co

** Especialista en Ciencias Políticas y Candidato a Magister en Educación. Profesor - investigador del Centro de Estudios de Didáctica y Pedagogia - CEDIP. Universidad de Ibagué. milton.trujillo@unibague.edu.co

Texto original recibido: $30-01-08$ y aprobado: 03-04-08

Pedagogía y Saberes N. ${ }^{\circ}$ 28. Universidad Pedagógica Nacional. Facultad de Educación, 2008, pp. 93-104 


\title{
REFLECTIONS UPON THE DIDACTICS OF THE SOCIAL SCIENCES: CONTRIBUTIONS FOR THE TEACHERS WHO GUIDE THE SOCIAL SCIENCES TEACHING IN THE ELEMENTARY AND MEDIUM EDUCATIONAL LEVELS
}

\begin{abstract}
The authors affirm that the purpose of the teaching of the social sciences is to form neither social scientists nor experts in a science or discipline. What it is taught in social sciences must exceed the knowledge about them and it's found tied to the social formation of the school so that children and adolescents have the necessary tools to read, to interpret, to analyze critically and to develop in the reality in which they are found immersed and to work to improve such reality. Hence, the one thing that interested to classical didactics, that was remitted to the methodological devices, should be redefined, since not only the postmodern condition requires to think about the information but the educational event implies other elements which had not been kept in mind, as the problem of the representations, of the transpositions, which exceed the problem of the conceptualization. With all, the interest of the authors of this text has been to discuss how, in the contemporary educational world, it is possible to think over the didactics of the social sciences, with new paradigms and new forms of teaching, adequate to the complexity of the present world and strengthening the emancipating and comprehensive purposes of the critical-social thought. In this order of ideas, it is fundamental that this text is a model of reflection for the teachers of social sciences about their educational practices and their formation to discipline.
\end{abstract}

Key words: Didactics, hermeneutics, critical thought, social sciences.

\section{INTRODUCCIÓN: EL CAMPO DE LAS CIENCIAS SOCIALES}

Tradicionalmente bajo el campo de las ciencias sociales se engloban los conceptos y problemas relacionados con la historia y la geografia, con algo de antropología y un conglomerado poco definible de cuestiones relacionadas con la formación civica, con la politica, la economia y hasta la urbanidad, lo que muestra que la denominación ciencias sociales se emplea para englobar contenidos que en muchos casos, no tienen claro su referente disciplinar $y$, en otros, mezclan informaciones diversas procedentes de estas disciplinas.

En el campo social, es aceptada sin discusión la idea de que la realidad existe objetivamente al margen de la propia voluntad, y que una parte de esa realidad global tiene unas caracteristicas particulares que son todas aquellas que se derivan de la presencia y acción de los seres humanos, y es a la que se denomina genéricamente "lo social". Este axioma precisa que "lo social" es un todo objetivo capaz de ser analizado y explicado unitariamente desde la diversa contribución de varias disciplinas (pun- tos de vista, concepciones, representaciones acudiendo a lo racional o a las sensibilidades) según el tema particular objeto de estudio. De ahí que es importante resaltar la pluralidad de visiones de "lo social", lo que implica necesariamente llamar a este saber ciencias sociales y no Ciencia (única y exclusiva) de "lo social". En otras palabras, la unidad de la realidad social se estudia desde la diversidad de las disciplinas concretas sin menoscabar a aquella, pues la unidad está en el objeto y la diversidad en los enfoques.

Las ciencias sociales se caracterizan por una pluralidad de métodos y esquemas conceptuales. Aunque todas procuran la comprensión y explicación de procesos y fenómenos sociales, cada una lo hace desde una óptica particular. Hasta cierto punto, pues, podríamos afirmar que los logros de una ciencia son el producto de la fragmentación de una misma realidad interpretada desde perspectivas independientes, si no fuera porque existe. de hecho, una interconexión y, más aún, una interdependencia entre las distintas disciplinas sociales (CAMILLONI y LeVINAs.2001p. 9).
En concreto, lo que las ciencias sociales han intentado hacer es profundizar en su carácter hermenéutico, con el fin de explicar cómo eventos principales o destacados de la historia, están relacionados entre ellos, a través de variables como tiempo y espacio, por ejemplo; han intentado, además, comprender y distinguir causa, efecto, secuencia y relación de eventos históricos, incluyendo relaciones causales a largo y a corto plazo, comparando el presente con el pasado, evaluando consecuencias y decisiones pasadas; para determinar lecciones aprendidas. En pocas palabras, se han interesado, estas Ciencias, por interpretar significados, implicaciones c impactos de eventos y fenómenos sociohistóricos

Todas las sociedades han asignado siempre enorme importancia al hecho de que las jóvenes generaciones conozcan las tradiciones, las experiencias colectivas y la organización y funcionamiento de la sociedad como forma de adquirir cierta identidad, haciendo surgir costumbres, normas, valores, simbolos comunes, es decir, ciertos modos de ver el mundo y de concebirse en él. Gran parte de la educación, en su función socializado- 
ra, ha consistido en conocer la propia sociedad, su pasado histórico, asi como el espacio y territorio en que se desarrolla la vida del grupo. En la sociedad moderna, la escuela es la institución encargada de asegurar esa función (SECRETARIA DE EDUCACIÓN DE LA Provincia de Buenos Aires, 2003).

Ahora bien, hoy un "nuevo" interés supera el de la explicación, pues una de las propuestas que ha ganado ventaja está dada por la comprensión de los hechos sociales, y su posible transformación, ya que la realidad es susceptible de cambio; asi, "las ciencias sociales son formas o estructuras para representar la interacción simbólica en el mundo de la vida, del mundo histórico-social" (VARGAS GUILLÉn y Rueda. 1996), y como tal, exigen pasar a un nivel de comprensión mucho mayor que el tratado tradicionalmente, un nivel de mayor complejidad, mayores relaciones en cantidad y cuali- dad sistémicas que evidencian la misma dinámica de lo social.

Las ciencias sociales no deben ser ni mero recuento de los hechos del pasado (historia tradicional), ni tampoco la simple búsqueda de regularidades con una visión ahistórica. [...] Si decidimos, por tanto, que la unidad de análisis no es ya el Estado-nación, sino el sistema-mundo debemos además acudir al análisis transdisciplinario eliminando la tradicional distinción entre el método de análisis idiográfico propio de la historia y el nomotético propio de la antropología, economía, ciencias políticas y sociología (LÓPEZ, 2000).

\section{PROBLEMAS Y \\ METODOLOGIAS DE LAS CIENCIAS SOCIALES}

Para abordar el problema de las ciencias sociales es fundamental comprender la naturaleza compleja de estos saberes y su inconmensurabilidad. En palabras de Llopis y Carral:

es evidente que las ciencias sociales se nos presentan como una pluralidad confusa y desordenada, sin un contenido perfectamente definido y con un origen y desarrollo particular de cada una de las disciplinas que la engloban. Entre todas tratan de dar una interpretación del hombre teniendo en cuenta su dimensión social, por eso las entendemos como un sistema interrelacionadas entre sí, nunca como una mera yuxtaposición enciclopédica (1986, pp. 18-19).

Desde esta perspectiva, abordar el fin de las ciencias sociales exige entender la complejidad no sólo de los hechos sociales, sino la complejidad y la dinámica interrelacional que tienen los aspectos que definen un problema, como se verá en el gráfico 1.

\section{Ciencias sociales

$\downarrow$ \\ Describen, explican y comprenden al hombre en relación consigo mismo, con otros y con su entorno local y global}
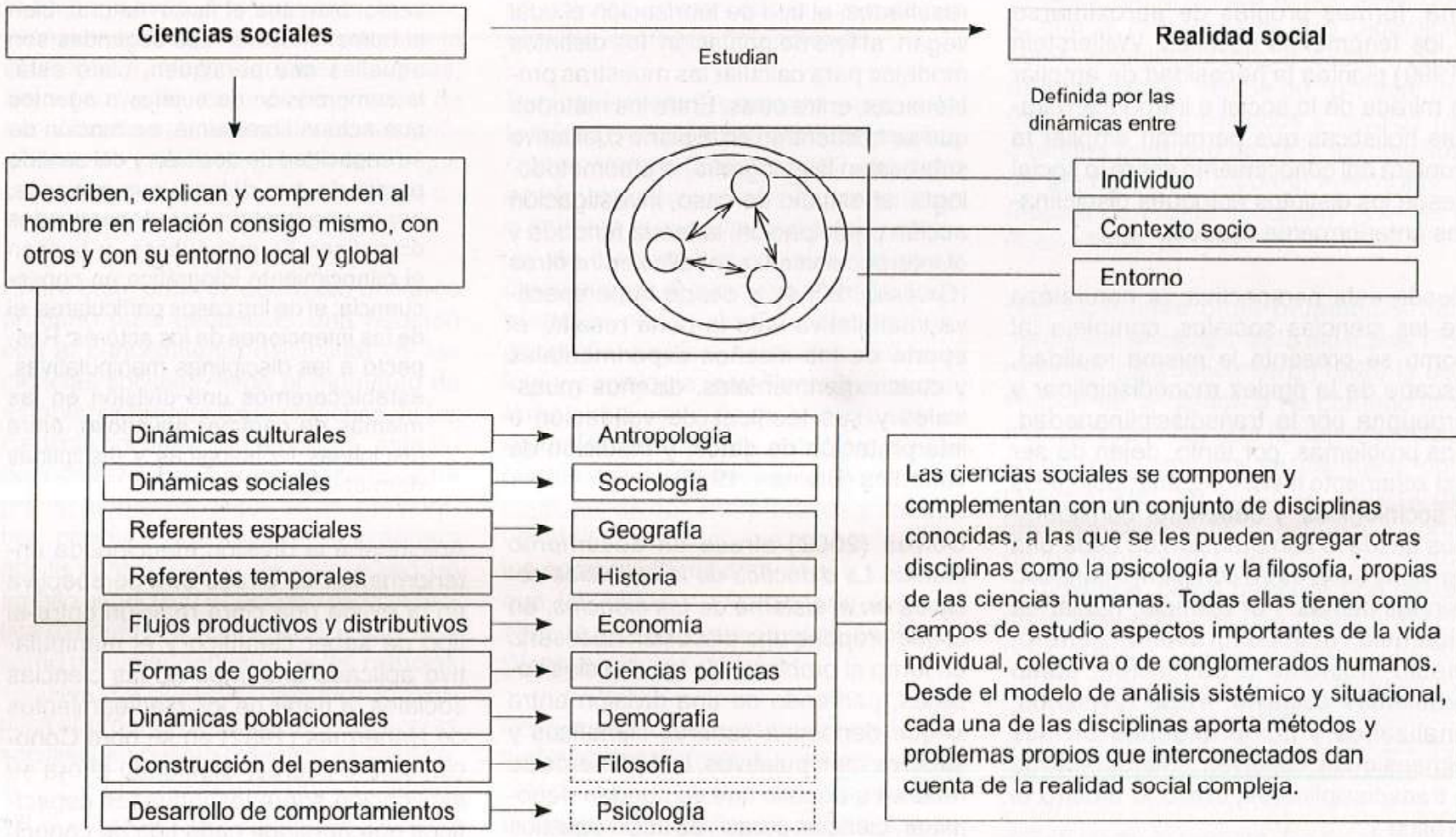

\begin{tabular}{|l|}
\hline Antropologia \\
\hline Sociologia \\
\hline Geografia \\
\hline Historia \\
\hline Economia \\
\hline Ciencias politicas \\
\hline Demografia \\
\hline Filosofia \\
\hline Psicologia \\
\hline
\end{tabular}

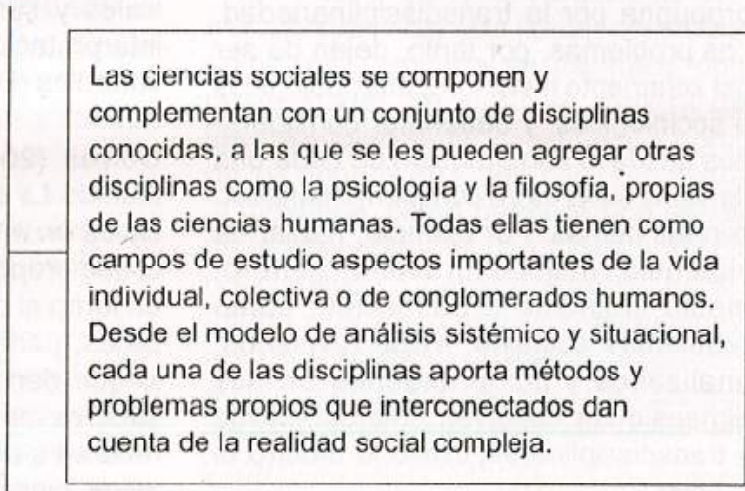

Gráfico 1

Permite distinguir la acción de las ciencias sociales en la comprensión de la realidad social y la compleja dinámica que tienen que asumir las disciplinas que constituyen este saber para validar su interés hermenéutico y crítico. Fuente: Gutiérrez y Trujillo (inédito). 
De la misma forma que se reconoce en las ciencias sociales su diversidad de posturas epistemológicas y teóricas, se hace fundamental reconocer los diferentes enfoques metodológicos que permiten al investigador realizar una aproximación al objeto mismo de conocimiento, que como se citó anteriormente, es el Hombre y sus múltiples interacciones con el entorno.

La caracterización de las ciencias sociales permitió, desde sus primeros desarrollos históricos, ser reflexionada como parte de la naturaleza y como construcción cultural. De ahi que fuera necesaria la búsqueda de formas metodológicas que permitieran su conocimiento; desde las perspectivas positivistas hasta las hermenéuticas (MARDONES, 1991.)

Para poder comprender la manera como las ciencias sociales abordan un problema será necesario dimensionar las perspectivas disciplinares implicitas; así, será posible reconocer en cada una, formas propias de aproximarse a los fenómenos sociales. Wallerstein (1999) plantea la necesidad de ampliar la mirada de lo social e introducir miradas holisticas que permitan ampliar la frontera del conocimiento sobre lo social desde los distintos enfoques disciplinares anteriormente citados.

Desde esta perspectiva, la naturaleza de las ciencias sociales, compleja tal como se presenta la misma realidad, escapa de la rigidez monodisciplinar y propugna por la transdisciplinariedad. Los problemas, por tanto, dejan de ser asi solamente históricos, antropológicos o sociológicos, y deben ser considerados desde la comprensión de cada una de estos saberes en complementariedad con los demás. Por ejemplo, hablar de violencia, multiculturalidad, género, medio ambiente o educación, como problemas sociales, exige revisarlos, analizarlos y comprenderlos en sus dimensiones objetivas, intersubjetivas y transdisciplinares, como lo mostró el gráfico 1.

El reconocer en las ciencias sociales la fuente de comprensión e interpretación de las realidades colectivas, permite el desarrollo de una serie de métodos que buscan aproximar al investigador al sujeto-objeto de estudio de forma tal que finalmente se pueda hablar de una realidad comprendida.

Las discusiones sobre "El" método cientifico han ido dejando atrás concepciones tales como el monismo metodológico, la necesidad de la explicación causal y la creencia de que el único lenguaje posible en la ciencia es el fisico-matemático debido a su intención de exactitud. Se acepta que un problema puede ser visto bajo distintas interpretaciones y que éstas pueden responder a intenciones particulares del conocimiento.

La complejidad de las ciencias sociales permite realizar estudios desde perspectivas cuantitativas y cualitativas. Se encuentran diferencias sustanciales entre los métodos cuantitativos y cualitativos en el orden de los problemas que estudian, las leyes que buscan (o no), en las formas de dar validez a sus resultados, al tipo de teorización al cual llegan, al tipo de población, los distintos modelos para calcular las muestras problémicas, entre otras. Entre los métodos que se encuentran en el plano cualitativo sobresalen la etnografia, la etnometodologia, el estudio de caso, investigación acción participación, la teoria fundada y el interaccionismo simbólico entre otros (GALEANO, 2004); y desde la perspectiva cuantitativa vale la pena resaltar el aporte de los diseños experimentales y cuasiexperimentales, diseños muestrales y sus técnicas de validación e interpretación de datos, y medición de variables (BRIONES, 1995)

Correa (2002) ofrece un documento titulado La didáctica de las ciencias sociales en el sistema de las ciencias, en el que propone una discusión necesaria en torno al problema de las ciencias sociales, partiendo de una división entre lo que denomina saberes cientificos y saberes manipulativos. Los primeros se refieren a aquello que se pueden denominar "ciencias puras", es decir, aquellos saberes que buscan exclusivamente el saber por el saber, el conocimiento por el conocimiento. Igualmente, se podrian denominar ciencias explicativo-com- prensivas, puesto que incluirian tanto a las ciencias que buscan la explicación de fenómenos como a aquellas que buscan su comprensión.

Con el nombre de disciplinas manipulativas hace referencia a aquellos saberes cuya pretensión estriba en establecer una modificación efectiva sobre el universo real. Es decir, que el ser humano, mediante ese conocimiento que producen o atesoran, de forma voluntaria y deliberada pueda alterar la realidad en un sentido previamente determinado; esto es, que permiten manipular la realidad en alguna medida con un sentido previo.

En las disciplinas cientificas se puede establecer una subdivisión respecto a la naturaleza del propio conocimiento entre ciencias explicativas y ciencias comprensivas, ateniéndonos a la clásica sistematización. Las primeras son las ciencias positivistas, es decir, aquellas que pretenden establecer de forma inequivoca las leyes generales que rigen el funcionamiento del universo, bien sea el fisico-natural, bien el humano-social. Las segundas son aquellas que persiguen, claro está, la comprensión de sujetos o agentes que actúan libremente, en función de su capacidad de decisión y del sentido propio de que dotan a sus acciones, por lo que también son denominadas disciplinas hermenéuticas, y buscan el conocimiento idiográfico en consecuencia: el de los casos particulares, el de las intenciones de los actores. Respecto a las disciplinas manipulativas, estableceremos una división en las mismas de carácter novedoso, entre disciplinas tecnológicas y disciplinas normativas.

Adicional a la división mencionada anteriormente, se ofrece otra perspectiva en la existe una clara relación entre el tipo de saber científico y el manipulativo aplicado al campo de las ciencias sociales, a partir de los planteamientos de Habermas (1982) en su obra Conocimiento e interés. Siguiendo ahora su explicación sobre los intereses especificos que persigue cada tipo de conocimiento, tenemos que el conocimiento positivista sigue un interés tecnológico; es decir, una vez conocidas las leyes que gobiernan un ámbito del mundo, 
es consecuencia la capacidad del ser humano para prever el desarrollo y final del proceso, e, interviniendo sobre las condiciones, conseguir un objetivo predeterminado. La disciplina tecnológica, no crea ciencia sensu stricto, sino que la utiliza, y su saber es el fruto de la aplicación del conocimiento de referencia a la realidad material. Eso es lo que Habermas, críticamente, denomina razón instrumental o técnica.

Por el lado de las ciencias idiográficas se establece que las guia un interés práctico. En cuanto que no establecen leyes de la realidad, a las que las cosas han de necesariamente plegarse y seguir, es imposible la manipulación científicotecnológica. En cambio, en cuanto las ciencias idiográficas (el conocimiento científico hermenéutico) proporciona un conocimiento sobre contextos particulares y sujetos, de tipo comprensivo, es posible aplicar ese saber de forma práctica en la realidad, pero basándose en la comprensión del sentido que todo lo humano tiene. Por supuesto, la comprensión es subjetiva, por tanto sujeta a errores e interpretaciones diversas, pero eso no le resta posibilidades de ser utilizada prácticamente (no tecnológicamente, desde luego). De forma que estas ciencias interpretativas tienen un interés que se denomina práctico.

Ahora bien, una vez abordado el marco primario para establecer una relación con el conocimiento disciplinar de las ciencias sociales, llega el momento de establecer el lugar de la didáctica de las ciencias sociales. Sin embargo, esta labor conlleva serios problemas desde un primer momento, desde su mismo nombre, porque exige plantear la pregunta por la consideración de ésta como un ámbito especifico y concreto de la didáctica (o como una aplicación), o asumirla como un saber derivado de las ciencias sociales. La primera consideración implica abordar la didáctica de las ciencias sociales, en cuanto didáctica especifica; la segunda consideración intenta repensar la Didáctica de las ciencias sociales en cuanto aborda el conocimiento de las ciencias sociales.

El primer intento de acercamiento de los autores del presente escrito, sobre el análisis de la Didáctica de las ciencias sociales se realizará acudiendo a la primera consideración.

\section{EL CAMPO DE LA DIDÁCTICA DE LAS CIENCIAS SOCIALES}

Con respecto a la Didáctica de las ciencias sociales se puede afirmar que los lineamientos más frecuentemente tomados en cuenta en la pregunta por el objeto y los problemas que debe abordar la enseñanza de las ciencias sociales en la Escuela, están diseñados por el Ministerio de Educación Nacional y han sido llamados "estándares", con la intensión de ser tomados como "una guia para que los alumnos de todos los niveles puedan dar una mirada al individuo en la sociedad y su relación con el medio ambiente a lo largo del tiempo". (Ministerio de Educación Nacional, 2004). Se resalta, por tanto, como objeto de estudio al hombre como ser que se relaciona con otros hombres y con un entorno, enmarcado en un contexto espacio-temporal determinado.

Para revisar esas relaciones y representaciones -empleando la conceptualización de Vargas Guillén (1996)- se debe acuidir también a diferentes disciplinas o saberes que comprenden el "universo" de las ciencias sociales, como se mencionó en el apartado anterior, comprendido principalmente por historia, geografia, politica, economia, antropología, sociología, psicología y filosofía, entre otras, con el fin de "comprender la naturaleza cambiante y relativa de los puntos de vista y entender que son susceptibles de ser interpretados y controvertidos", a la vez que se intenta "asumir los compromisos personales y sociales que los niños y jóvenes adquieren a medida que avanzan en el aprendizaje" (VARGAS Y RUEDA, 1996.)

Un ejemplo que puede orientar la actual discusión sobre el problema de la didáctica en ciencias sociales es aportado por la Secretaria de Educación de la Provincia de Buenos Aires, Argentina (2003):

la finalidad de la enseñanza de las ciencias sociales no es formar cien- tificos sociales ni expertos on una ciencia o disciplina. Lo que se enseña en ciencias sociales debe rebasar el conocimiento de las mismas y se encuentra ligado a la formación social de la escuela para que niños y adolescentes tengan las herramientas necesarias para leer, interpretar, analizar criticamente y desarrollarse en la realidad en la que se hallan inmersos y trabajar para mejorarla.

Asi, pues, aquello que le interesaba a la Didáctica Clásica (con pretensiones de generalidad) que se remitia a los dispositivos metodológicos debe ser replanteado, pues no sólo la condición postmoderna (Lyotard, citado por VARGAS y RUFDA, 1996, p. 172) exige pensar en la información (en oposición a la razón ilustrada) sino que el acontecimiento educativo implica otros elementos que no se habian tenido en cuenta, como el problema de las representaciones, de las transposiciones, que superan el problema de la conceptualización. Los autores son enfáticos al concretar que lo que le corresponde a la Didáctica, como "acontecimiento" y "espacio de la representación" reside en "plantear las estrategias, los métodos y medios requeridos para que se de la apropiación de simbolos, de valores, ideologias y utopias dentro de la cultura" (VARGAS y RUEDA, 1996, p. 177)

Históricamente otras propuestas han dominado el campo de la didáctica. Por ejemplo, para Aisemberg (1996) la definición de la didáctica ha contado con tres perspectivas que la han considera como una disciplina no cientifica, con posibilidades de serlo; la primera de las perspectivas se constituye a partir de los aportes de Camilloni, pạra quien la didáctica, como parte de una teoría de la educación, es una teoría práctica no-cientifica. Chevallard, por su parte, afirma que las didácticas especificas son disciplinas que al tener investigación empírica de la enseñanza en un campo determinado se pueden constituir en disciplinas cientificas, ya que su estado actual no se lo permite. En tercer lugar, para Brousseau, entre otros, la didáctica es un conjunto de reflexiones, suma de diversos saberes científicos que al no tener investigaciones especificas ni 
conceptos propios no puede ser reconocida como ciencia. Ahora bien, aplicando las conceptualizaciones mencionadas al campo de la didáctica de las ciencias sociales se puede afirmar que ésta se encuentra muy lejos de poseer una teoria especificamente didáctica que de cuenta de investigación sobre el objeto propio debido a la multiplicidad de disciplinas que la constituye y a la falta de reflexión didáctica sobre cada una.

A partir de la conceptualización realizada en la primera parte del escrito, en donde se resalta la necesidad de la interdisciplinariedad y transdisiplinariedad en la tarea de explicar y comprender los fenómenos sociales (ya que la realidad en si misma es compleja), se encuentra que el enfoque de la didáctica de las ciencias sociales exige lo mismo, pues no es pertinente plantear la necesidad de didácticas especificas para cada disciplina que constituyen las ciencias sociales, sino que se pueden unificar esos aportes con una sola mirada integradora; asi, la enseñanza de las ciencias sociales, de los problemas sociales, tendria una perspectiva complementaria, integradora y adecuada, según la propuesta de Aisemberg (1996). Asi, para dar cuenta de los problemas que atraviesan las ciencias sociales, desde la enseñanza, es necesario reforzar y resaltar la mirada transdisciplinar, evitando la fragmentación y la explicación monodisciplinar.

\section{PROBLEMAS $Y$ METODOLOGÍAS EN LA DIDÁCTICA DE LAS CIENCIAS SOCIALES}

Para abordar los problemas que constituyen la didáctica de las ciencias sociales es fundamental partir de una estructuración de los mismos. Para mayor comprensión se han dividido a partir de los siguientes elementos: un primer nivel de problemas está vinculado a la dificultad de identificar los marcos de referencia sobre los cuales gire la comprensión de la naturaleza de la didáctica específica. Un segundo nivel se refiere a los problemas propios de la relación conocimiento-profesor-estudiantes enmarcada en un contexto sociocultural particular.
Frente al problema de los marcos de referencia, diremos que comprende las imprecisiones conceptuales que han dificultado la construcción de una idea clara sobre la naturaleza de la didáctica de las ciencias sociales. Así, los marcos de referencia fragmentarios (multidisciplinares) no permiten abarcar la totalidad del objeto propio y limitan la reflexión acerca de la enseñanza, objeto último de la didáctica.

La enseñanza por ser un fenómeno social complejo no permite ser abordada desde una sola perspectiva disciplinar ya que lo que es evidente para una disciplina pasa desapercibido por otra, generándose asi, vacios conceptuales dificiles de identificar. El status de la didáctica se apoya, en la mayoria de los casos en los logros disciplinares y no en la reflexión sobre las prácticas pedagógicas. Por último, un problema complejo es la incoherencia epistemológica derivada de la fragmentariedad de los marcos teóricos que retroalimentan las disciplinas propias que conforman el corpus de la didáctica de las ciencias sociales.

En segundo lugar, los problemas de la didáctica de las ciencias sociales devienen de la complejidad de dinámicas presentes en la triada conocimiento-profesor-estudiante. Es necesario precisar el rol del profesor en el proceso de la enseñanza y del aprendizaje: La labor del profesor debe ser considerada a partir de los siguientes aspectos: Sus prácticas pedagógicas, la problematización de los contenidos disciplinares y la reflexión permanente sobre el proceso de formación (Compagnucci et ál., 2002).

En los ochentas, Elbaz propone que el saber de los profesores es un conjunto de conocimientos complejos, orientados a las prácticas que éstos utilizan activamente para conformar y dirigir el trabajo de la enseñanza. A partir de esta precisión construye cinco categorias de análisis de los problemas relacionados con sus prácticas: el conocimiento de si mismo (es decir, los valores implicitos), el conocimiento del medio (los espacios en los cuales lleva a cabo sus prácticas), el conocimiento de la asignatura (contenidos), conocimiento del curriculum (poli-

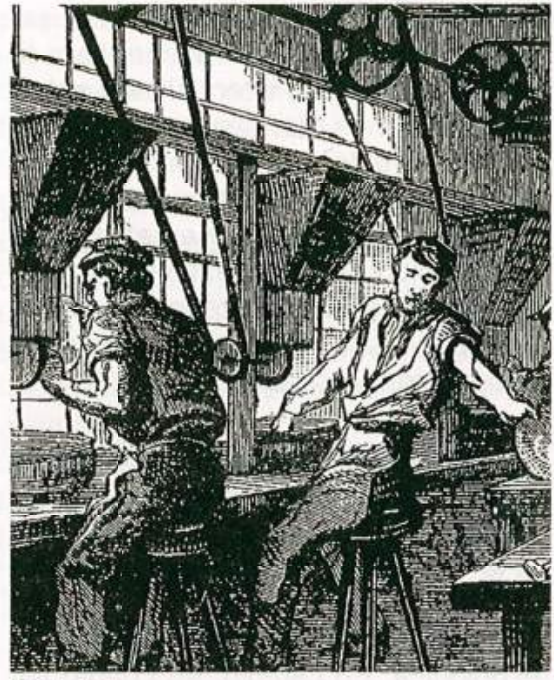

ticas) y el conocimiento de la instrucción (es decir, los enfoques teóricos desde los cuales piensa la enseñanza).

Los problemas que se evidencian en la práctica de la labor docente han sido clasificados de la siguiente manera:

- Relacionados con la formación: La mayoria de los profesores de las ciencias sociales son profesionales en diferentes disciplinas que carecen de una formación en pedagogía.

- Relacionados con las prácticas pedagógicas: Dificultades del docente en relación con sus propios conocimientos y por consiguiente imposibilidad de llevar a cabo la transposición didáctica que permite trascender del conocimiento disciplinar al conocimiento formativo. Falta de espacios adecuados para la reflexión pedagógica.

- Relacionados con el contexto institucional: Marginalidad de las ciencias sociales frente a otras disciplinas. Tradición de la enseñanza de la historia y la geografia como medio por excelencia de conocimiento social. Falta de politicas y programas de formación permanente.

Las dinámicas problematizantes manifiestas en las relaciones -conocimientoprofesor-estudiante son caracterizadas en las tablas 1 y 2 , adecuados para la reflexión de lo general a lo particular, como se muestra a continuación. 


\begin{tabular}{|c|c|c|}
\hline Reforencias & Temáticas de estudio & Preguntas de ciencias sociales \\
\hline $\begin{array}{l}\text { Temáticas de estudio } \\
\text { relativas a la enseñanza } \\
\text { de una disciplina } \\
\text { particular de referencia. }\end{array}$ & $\begin{array}{l}\text { - Laturaleza de la disciplina de referencia. } \\
\text { referencia. } \\
\text { - Las prácticas de enseñanza de la- disciplina de } \\
\text { referencia y sus efectos. } \\
\text { - Creencias y concepciones de los profesores sobre } \\
\text { el saber, la enseñanza y el aprendizaje de la } \\
\text { disciplina de referencia. } \\
\text { - El conocimiento de los profesores (de la disciplina } \\
\text { y didáctico) y su impacto. } \\
\text { - Los procesos de enseñanza en grupos en el aula } \\
\text { de clase. } \\
\text { - El conocimiento profesional de los profesores de } \\
\text { la disciplina de referencia. } \\
\text { - La formación inicial de los profesores de la } \\
\text { disciplina de referencia. } \\
\text { - Uso de la tecnologia en la enseñanza de la } \\
\text { disciplina de referencia. } \\
\text { - La relación: historia de los conceptos con su } \\
\text { enseñanza. } \\
\text { - La disciplina de referencia asumida como un } \\
\text { lenguaje especifico y sus efectos sobre la } \\
\text { enseñanza. } \\
\text { do virtual en los procesos de enseñanza de la } \\
\text { discina de referencia. }\end{array}$ & $\begin{array}{l}\text { - ¿Cuáles son las concepciones de Naturaleza de la } \\
\text { didáctica en ciencias sociales en la escuela? } \\
\text { ¿Cuál es el impacto de la cultura en la enseñanza } \\
\text { de las ciencias sociales? } \\
\text { ¿Qué relación existe entre las prácticas de } \\
\text { enseñanza de las ciencias sociales y sus efectos } \\
\text { en el contexto social? } \\
\text { - ¿Qué tipo de concepciones tienen los profesores } \\
\text { sobre el saber, la enseñanza y el aprendizaje de } \\
\text { las ciencias sociales en torno a los problemas } \\
\text { sociales? } \\
\text { - ¿Cuáles son los procesos de enseñanza que se } \\
\text { emplean para el abordaje de problemas sociales } \\
\text { desde las ciencias socialos? } \\
\text { - ¿Cuánto conocen los profesores de ciencias } \\
\text { sociales sobre su propia disciplina? } \\
\text { ¿Cuáles son los contenidos disciplinares con } \\
\text { los que ese forma a los profesores de ciencias } \\
\text { sociales? } \\
\text { - ¿Qué conceptos propios de la disciplina se } \\
\text { enseñan en las clases de ciencias sociales? } \\
\text { ¿De qué manera el empleo de lo virtual favorece } \\
\text { la enseñanza de las ciencias sociales? }\end{array}$ \\
\hline $\begin{array}{l}\text { Temáticas relativas al } \\
\text { aprendizaje de cada } \\
\text { uno de los conceptos o } \\
\text { grupos de conceptos de } \\
\text { una disciplina particular } \\
\text { de referencia }\end{array}$ & $\begin{array}{l}\text { - Aprendizaje de un(os) conceptos. } \\
\text { disciplina de referencia. } \\
\text { - Creencias y concepciones de los y las estudiantes } \\
\text { sobre el aprendizaje de la disciplina de referencia. } \\
\text { - Aprendizajo de los campos conceptuales } \\
\text { particulares. } \\
\text { - La transición de un nivel de complejidad } \\
\text { conceptual al siguiente. } \\
\text { - Niveles investigativos en cada campo conceptual } \\
\text { y direccionamientos de la investigación en ese } \\
\text { campo particular. } \\
\text { - Relaciones entre campos conceptuales diferentes } \\
\text { relativos a su aprendizaje. } \\
\text { - Construcción de conocimiento, pensamiento y } \\
\text { actitud relativos a la disciplina de referencia. }\end{array}$ & $\begin{array}{l}\text { - ¿Qué concepciones tienen los estudiantes sobre } \\
\text { las ciencias sociales y su aprendizaje? } \\
\text { ¿Qué direccionamientos de la investigación } \\
\text { existen en la actualidad para el desarrollo de los } \\
\text { procesos de aprendizaje de las ciencias sociales? } \\
\text { - ¿Cuáles relaciones existen entre las diferentes } \\
\text { disciplinas que componen las ciencias sociales en } \\
\text { torno a su aprendizaje? } \\
\text { ¿Qué relación puede establecerse entre saberes } \\
\text { conceptuales y actitudinales en la enseñanza de } \\
\text { ciencias sociales? } \\
\text { ¿Qué obstáculos epistemológicos entorpecen los } \\
\text { procesos de aprendizaje de las ciencias sociales? } \\
\text { ¿Qué esquemas de abordaje y resolución de } \\
\text { problemas existe en las ciencias sociales para } \\
\text { favorecer los procesos de aprendizaje de las } \\
\text { mismas? }\end{array}$ \\
\hline
\end{tabular}




\begin{tabular}{|c|c|c|}
\hline & $\begin{array}{l}\text { - Uso de la tecnologia en el aprendizaje de la } \\
\text { disciplina de referencia. } \\
\text { - Relación entre la historia de los conceptos y su } \\
\text { aprendizaje, obstáculos epistemológicos } \\
\text { - La disciplina de referencia .asumida como } \\
\text { un lenguaje especifico y sus efectos sobre el } \\
\text { aprendizaje. } \\
\text { - Esquemas en resolución de problemas de la } \\
\text { disciplina de referencia. }\end{array}$ & \\
\hline $\begin{array}{l}\text { Relativos a aspectos } \\
\text { variados de la } \\
\text { enseñanza y el } \\
\text { aprendizaje }\end{array}$ & $\begin{array}{l}\text { - El niño talento en la disciplina de referencia. } \\
\text { pognición en etnias particulares de una disciplina } \\
\text { - El afecto en enseñanza y aprendizaje de una disci- } \\
\text { plina particular de referencia. } \\
\text { - Relaciones entre aprendizaje y género. } \\
\text { - Evaluación de los aprendizajes sobre un conoci- } \\
\text { miento particular de referencia. } \\
\text { - Relaciones entre raza, clase social, lenguaje y con- } \\
\text { secución de logros y competencias en la disciplina } \\
\text { de referencia. } \\
\text { - Cantidad de conocimiento de la disciplina de referen- } \\
\text { cia que debe poseer un profesor de tal disciplina. } \\
\text { - Relaciones entre el conocimiento particular de una } \\
\text { disciplina de referencia y su puesta en escena en un } \\
\text { contexto determinado para provocar el aprendizaje. } \\
\text { - El sentido constructivo del curriculo de una disci- } \\
\text { plina de referencia y su relación con los niveles de } \\
\text { aprendizaje. } \\
\text { - La interdisciplinariedad en la onseñanza y el apren- } \\
\text { dizaje de un conjunto conveniente de disciplinas de } \\
\text { referencia. } \\
\text { - Análisis de textos cientificos y análisis de libros de } \\
\text { texto empleados en el aula de clase. } \\
\text { - Relaciones entre la disciplina de referencia, su } \\
\text { enseñanza y su aprendizajo en la construcción de } \\
\text { competencias ciudadanas. } \\
\text { - Felaciones entre aprendizajes y contextos. } \\
\text { Fenomenologia didáctica de los conceptos. }\end{array}$ & $\begin{array}{l}\text { - ¿Cómo enseñar ciencias sociales en contextos } \\
\text { particulares como resguardos indígenas, población } \\
\text { vulnerable, etc? } \\
\text { - ¿De qué manera el afecto condiciona los procesos de } \\
\text { enseñanza y aprendizaje de las ciencias sociales? } \\
\text { - Tiene alguna implicación en la enseñanza de las } \\
\text { ciencias sociales los problemas de género } \\
\text { - ¿Cómo evaluar los aprendizajes sobre los conoci- } \\
\text { miento de historia, geografia, por ejemplo, desde la } \\
\text { integralidad de las ciencias sociales? } \\
\text { - ¿Cómo influyen aspectos como raza, clase social, } \\
\text { lenguaje y consecución de logros y competencias } \\
\text { en el desarrollo de contenidos de ciencias sociales } \\
\text { en la escuela? } \\
\text { - ¿Qué tipo de curriculo orienta el desarrollo de las } \\
\text { clases de ciencias sociales en la escuela? } \\
\text { - ¿Cómo se concibe y se trabaja en torno a la inter- } \\
\text { disciplinariedad en la enseñanza y el aprendizaje de } \\
\text { las ciencias sociales? } \\
\text { - ¿Qué tipos de textos y libros de texto se empleados } \\
\text { en el aula de clase para la enseñanza de las ciencias } \\
\text { sociales? } \\
\text { - ¿Qué relación existe entre la enseñanza de las } \\
\text { ciencias sociales y la formación de competencias } \\
\text { ciudadanas? } \\
\text { enseñando en ciencias sociales, lo aprendido en } \\
\text { clase y el contexto social? }\end{array}$ \\
\hline
\end{tabular}

Tabla 1.

Temáticas relacionadas a la didácticas de las ciencias sociales; construido a partir de las orientaciones del profesor Villarraga, Miguel en su programa de asignatura Problemas de la Educación y la Pedagogia II de la Maestria en Educación de la Universidad del Tolima. 2007. 


\begin{tabular}{|c|c|c|}
\hline Referencias & Problemas identificados & Preguntas asociadas \\
\hline $\begin{array}{l}\text { Relación profesor/ } \\
\text { contenidos }\end{array}$ & $\begin{array}{l}\text { - Reproducción de textos. } \\
\text { - Sobrevaloración de lo formal. } \\
\text { - Falta de construcción de conceptos. } \\
\text { - Falta de rigurosidad teórica. } \\
\text { - Transmisión expositiva. }\end{array}$ & $\begin{array}{l}\text { ¿Cómo se articulan los contenidos y metodologias } \\
\text { para desarrollar estrategias para la enseñanza- } \\
\text { aprendizaje? }\end{array}$ \\
\hline $\begin{array}{l}\text { Relación Profesor/ } \\
\text { estudiantes }\end{array}$ & $\begin{array}{l}\text { - En su mayoría las actividades son propuestas por } \\
\text { el profesor, desestimulando la participación del } \\
\text { estudiante. } \\
\text { - Estilos autoritarios }\end{array}$ & $\begin{array}{l}\text { ¿Qué expectativa tiene el estudiante con respecto } \\
\text { al conocimiento social y cómo lo relaciona con su } \\
\text { mundo y pensamiento social? }\end{array}$ \\
\hline $\begin{array}{l}\text { Relación Estudiante/ } \\
\text { contenidos }\end{array}$ & $\begin{array}{l}\text { Dificultad de interpretar los fenómenos sociales. } \\
\text { Desconocimiento de las ideas previas con las } \\
\text { cuáles los estudiantes podrian relacionar los } \\
\text { conceptos. } \\
\text { - No existe interrogación por parte de los } \\
\text { estudiantes de los problemas tratados. } \\
\text { - Aprendizaje basado en la reproducción del texto. } \\
\text { - Dificultad de construcción del conocimiento sobre } \\
\text { la complejidad de la naturaleza humana y social }\end{array}$ & $\begin{array}{l}\text { ¿Es posible comprender y relacionar los } \\
\text { contenidos con problemas especificos de la } \\
\text { realidad del estudiante? }\end{array}$ \\
\hline
\end{tabular}

Tabla 2.

Problemas asociados a la didáctica de las ciencias sociales.

Ahora bien, una vez identificadas las preguntas que orientan el replanteamiento de la didáctica de las Ciencias sociales, es necesario comprender los diferentes métodos a través de los cuales se pueden llegar a la solución de tales preguntas. En este campo es fundamental reconocer que los estudios en didácticas de las ciencias sociales se encuentran atrasados con respecto al de las otras disciplinas. La búsqueda de alternativas didácticas en esta disciplinas es motivo urgentes investigaciones por parte de los especialistas.

Sin embargo, para abordar los métodos empleados en el desarrollo de estrategias didácticas en las ciencias sociales, es necesario identificar algunas teorias referidas a cómo optimizar el desempeño de estudiantes y profesores en el lugar donde se produce el conocimiento.

Unas teorías propenden por la necesidad de identificar la manera como cada individuo adquiere y representa un conocimiento, desde la perspectiva de Gardner sobre Comprensiones Intuitivas de escolares. La estrategia desde este lugar es que el estudiante debe conocer de primera mano un problema para transformar a partir de la contrastación prejuicios e imaginarios. Es necesario además, ver al problema desde múltiples perspectivas teóricas y a partir de ahi construir nuevas representaciones teóricas. Es decir, bajo esta perspectiva se privilegia la comprensión del contexto social, sin la cual, se obstaculiza la comprensión de ciertas realidades (RUEDA y VARGAS, 1996)

Otra posibilidad metodológica nos remite a la teoria de cambio conceptual abordada por Carretero, desde concepciones previas de un hecho particular, la confrontación de estas concepciones con referentes más teóricos buscará la construcción de nuevos conceptos. Las anteriores junto a la Teoría de si- tuaciones didácticas buscan enfrentar el problema de la enseñanza a través de perspectivas constructivistas (RUEDA y VARGAS, 1996)

La Teoria Constructivista es el elemento mediador entre la conducta del profesor y los resultados del aprendizaje. Aqui el conocimiento es construido por el sujeto cuando interactúa con el medio y trata de comprenderlo. Esta teoría está sustentada por otras que la dinamizan: La Teoria epistemológica de Piaget sobre el conocimiento como construcción y sus niveles de desarrollo cognitivo, teoría del Aprendizaje verbal significativo de Ausubel y la teoria del origen sociocultural de los procesos psicológicos superiores de Vigotsky (LLopIS, 2003). Llopis propone que para transformar el conocimiento común en conocimiento conceptual o científico es necesario comprender las culturas experienciales de estudiantes y profesores. 


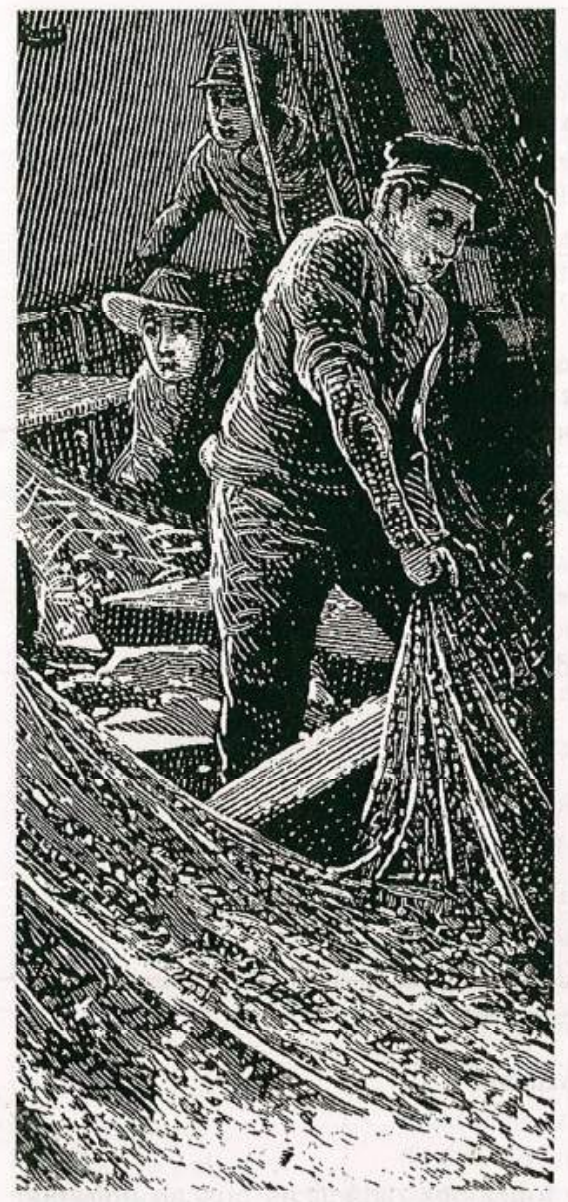

\section{COROLARIO}

Asi, pues, desde la mirada que hemos lanzado a las ciencias sociales, y principalmente a su didáctica, consideramos que estả reflexión entraña una ampliación, reconfiguración y actualización de los distintos campos del conocimiento no sólo de la disciplina como tal, sino de los docentes, en torno a utilización de tradicionales y nuevos recursos y las estrategias metodológicas para lograr una adecuada transposición al aula.

Es decir, luego de tener claros los referentes anteriores, podemos iniciar a abrir un espacio de reflexión ulterior con un especto de preguntas que, desde la conceptualización de de Pagés, podrian dar luces sobre:
Para Marrero (1993), estas experiencias son teorias reconstruidas sobre la base del conocimiento pedagógico históricamente elaboradas y transmitidas a través de la formación y práctica pedagógica. Por otra parte, Domenech (1999) afirma que son sólidas opiniones que nos formamos en función de nues-

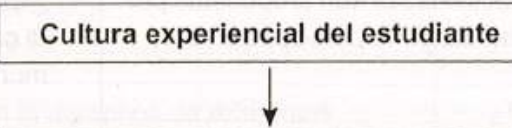

$$
\begin{aligned}
& \text { - Conocimientos previos } \\
& \text { - Creencias } \\
& \text { - Expectativas } \\
& \text { - Actitudes }
\end{aligned}
$$

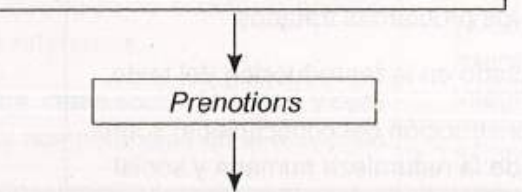

tra experiencia previa, nuestras teorias personales sobre la enseñanza y el aprendizaje (Lı_opIS 2003).

En breve, el gráfico 2 contribuye a la comprensión de esta propuesta constructivista.

A través del constructivismo se transforman los conocimientos previos en conocimiento cientifico.

Gráfico 2.

Constructivismo y conocimiento científico.

¿Para qué, qué y cómo enseñamos geografia, historia y otras disciplinas sociales? ¿Qué problemas hallamos en su enseñanza? ¿Aprenden realmente los alumnos aquello que les enseñamos con nuestras frases? ¿Qué necesita saber y saber-hacer un profesor o una profesora para enseñar geografia, historia y otras disciplinas de ciencias sociales? ¿Necesita saber y saber-hacer lo mismo el profesor de primaria y de secundaria o el universitario? ¿Quién prepara al profesorado de geografia o historia para enseñar? ¿Cómo se le prepara?

Finalmente, entonces, en términos prácticos diremos que resulta necesario al momento de reflexionar sobre los procesos de enseñanza y aprendizaje de las ciencias sociales, para poder iniciar una planeación concreta en el aula de clase:

- Una selección pertinente de contenidos (conceptuales, procedimentales $y$ actitudinales) sin perder de vista la adecuación, según el tema o problema que se aborda, con el contexto local y global.

- Una organización y jerarquización de los contenidos a enseñar: definir qué enseñar (determinar los tiempos y espacios que permitirán desarrollar los contenidos y los conceptos sobre los que se profundizará en el trabajo escolar) y cuándo hacerlo (cómo distribuirlos a lo largo de los años de formación; cómo lograr una coordinación de los mismos en los diversos ciclos de formación; cómo 
distribuirlos en el transcurso del año escolar).

- Una metodologia de enseñanza en el área, determinar qué recursos y estrategias utilizar.

- Una evaluación que incluya los diferentes momentos del proceso de aprendizaje: diagnóstico, formativo y sumativo.
Si estructuramos las preguntas en torno a los momentos o referentes importantes de ia clase, a partir de las orientaciones reflexivas de la Secretaría de Educación de la Provincia de Buenos Aires, Argentina. 2003, resulta como instrumento orientador la tabla 3 que aparece a continuación.

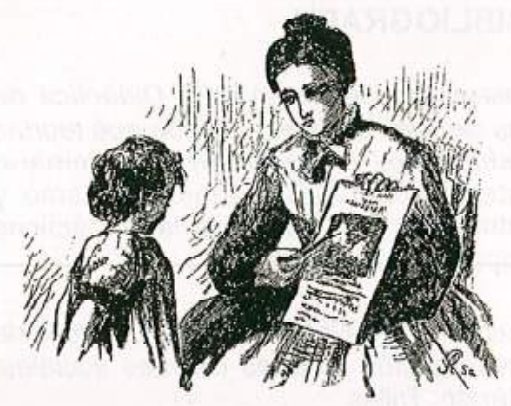

\begin{tabular}{|c|c|}
\hline Al comenzar la clase: & $\begin{array}{l}\text { ¿La clase se encuentra incluida en el trabajo global del curso? ¿Se retoma una temática iniciada } \\
\text { previamente, sintetizando lo realizado hasta el momento? ¿Se propone una nueva temática desconectada } \\
\text { del trabajo anterior? ¿Se parte del planteo de una situación problemática o del análisis de un caso? }\end{array}$ \\
\hline $\begin{array}{l}\text { Con respecto a los } \\
\text { contenidos }\end{array}$ & $\begin{array}{l}\text { ¿Se presenta un tiempo lineal - cronológico o se favorece el trabajo a partir de periodizaciones } \\
\text { posibilitando la comprensión de la simultaneidad y la sucesión, sincronia (se estudian las sociedades } \\
\text { identificando sus rasgos económicos, políticos, tecnológicos, culturales, en el contexto de una época } \\
\text { determinada) - diacronia (se promueven las capacidades de representación y medida del tiempo, } \\
\text { identificación de factores de cambio y permanencia y de las repercusiones de los acontecimientos, } \\
\text { coyunturas y procesos a corto, mediano y largo plazo)? } \\
\text { ¿Se favorecc la comprensión de las rolaciones entre la sociedad y el medio natural? ¿Se permite } \\
\text { establecer relaciones (dependencia, complementariedad) entre diversas escalas de análisis? ¿Se } \\
\text { favorece la comprensión del espacio como construcción social? } \\
\text { ¿Se presentan sujetos únicos individuales/colectivos o multiplicidad de ellos? ¿Se permite que los } \\
\text { alumnos trabajen a partir de diferentes puntos de vista de los sujetos sociales y que se manifiesten los } \\
\text { intereses que éstos expresan? ¿Se posibilita que se descubran las relaciones de poder existentes en una } \\
\text { sociedad y las situaciones de conflicto-consenso que en ella se desarrollan? }\end{array}$ \\
\hline $\begin{array}{l}\text { Con respecto a la } \\
\text { metodologia de trabajo }\end{array}$ & $\begin{array}{l}\text { ¿Se parte de los conocimientos previos de los alumnos? } \\
\text { ¿Se establecen relaciones con los contenidos trabajados previamente? } \\
\text { ¿Se generan estrategias para que los alumnos puedan aproximarse a la construcción de conceptos o se } \\
\text { proponen aprioristicamente definiciones acerca de los conceptos trabajados? } \\
\text { ¿Se dedica tiempo a escuchar las argumentaciones y explicaciones que dan los alumnos en relación a los } \\
\text { temas tratados? } \\
\text { ¿Se combinan instancias expositivas con instancias de trabajo individual o grupal? Si alienta el trabajo } \\
\text { individual o grupal ¿controla el progreso o estancamiento del mismo? } \\
\text { ¿Se utiliza sólo el texto como recurso o se combina el trabajo con el aporte de otras fuentes y otros } \\
\text { materiales? } \\
\text { ¿Se proponen situaciones problemáticas, explicaciones de conflictos, interpretación de historias de } \\
\text { vida, realización de predicciones, preparación de debates? ¿Se incluyen los pasos de la investigación } \\
\text { social (delimitación del problema, planteo de hipótesis. búsqueda y análisis de fuentes, elaboración de } \\
\text { conclusiones, comunicación de los resultados de la investigación por distintas vias)? } \\
\text { ¿Se generan instancias para que los alumnos reflexionen acerca sus procesos de aprendizaje? } \\
\text { ¿Se confrontan las ideas previas con la nueva información a la que se ha accedido? }\end{array}$ \\
\hline Al terminar la clase & $\begin{array}{l}\text { ¿Se plantea un cierre (integración o sintesis) de los contenidos abordados y evalúa lo desarrollado? } \\
\text { ¿Se realiza una revisión y reconstrucción del proceso de conocimiento realizado? }\end{array}$ \\
\hline
\end{tabular}

Tabla 3.

Sintesis reflexiva. 


\section{BIBLIOGRAFIA}

Aisemberg, Beatriz (1996). Didáctica de las ciencias sociales: ¿desde qué teorías estudiamos la enseñanza? Seminario internacional sobre constructivismo y educación en Relación a las didácticas especiales. Uruguay.

Briones, G (1995) Métodos y Técnicas de Investigación para las ciencias sociales. México: Trillas.

Camilloni A. W y Levinas M. L (2001). Pensar, Descubrir y Aprender: Propuesta didáctica y Actividades para las ciencias sociales. Buenos Aires: Aique.

Compagnucci, Elsa et ál (2002). Acerca de las prácticas docentes y la enseñanza de la psicología. En: Revista de Teoria y Didáctica de las ciencias sociales. Mérida, Venezuela. enero-diciembre. $\mathrm{N}^{0} 7$. p. 7-24.

Correa López, M. J. La Didáctica de las ciencias sociales en el sistema de las ciencias. Propuesta para una discusión necesaria. En: http://www.unizar.es/cuadernos $/$ n04/n04a02.pdf

Galeano, M. E (2004). Estrategias de Investigación Social Cualitativa: El giro de la mirada. La Carreta, Medellin.

http://abc.gov.ar/lainstitucion/sistemaeducativo/diepregep/capacitacion/cuadernillos/cuadernillo5.doc consultada el 17 de Febrero de 2007.

LLopIS, Ramón (2003). La docencia de la Sociologia y la cultura experiencial previa de alumnos y profesores. En: Revista de Teoria y Didáctica de las ciencias sociales. Mérida, Venezuela. enero-diciembre. $\mathrm{N}^{\circ}$ 8: p. 105-124.

LÓPEZ Segrera, F (2000). Abrir, impensar, y redimensionar las ciencias sociales en América Latina y el Caribe ¿Es posible una ciencia social no eurocéntrica en nuestra región? En http://www.clacso.org/wwwclacso/espanol/html/libros/lander/9.pdf. Consultada el 21 de abril de 2007
MARDONES, J.M (1991). Filosofía de las Ciencias Humanas y Sociales: materiales para una fundamentación científica. Bogotá: Anthropos,

Ministerio de Educación Nacional (2004). Estándares de ciencias Sociales. En: http:/l menweb.mineducacion.gov.co/prensa/ detallenoticias . asp? offset $=400$ yid $=56$ consultada el 17 de febrero de 2007

PAGÉs, J (1994). La didáctica de las ciencias sociales, el currículum y la formación del profesorado, En Signos. Teoría y practica de la educación año 5 - Número 13- octubre diciembre 1994.

VARGAS, G. y RUEDA, R (1996). Los Hipertextos como Didáctica en la Enseñanza de las ciencias sociales, en Simposio Latinoamericano de Didáctica de las Disciplinas de la Educación Básica.

Wallerstein, l. et ál. (1999). Abrir las ciencias sociales. Editorial Siglo XXI.

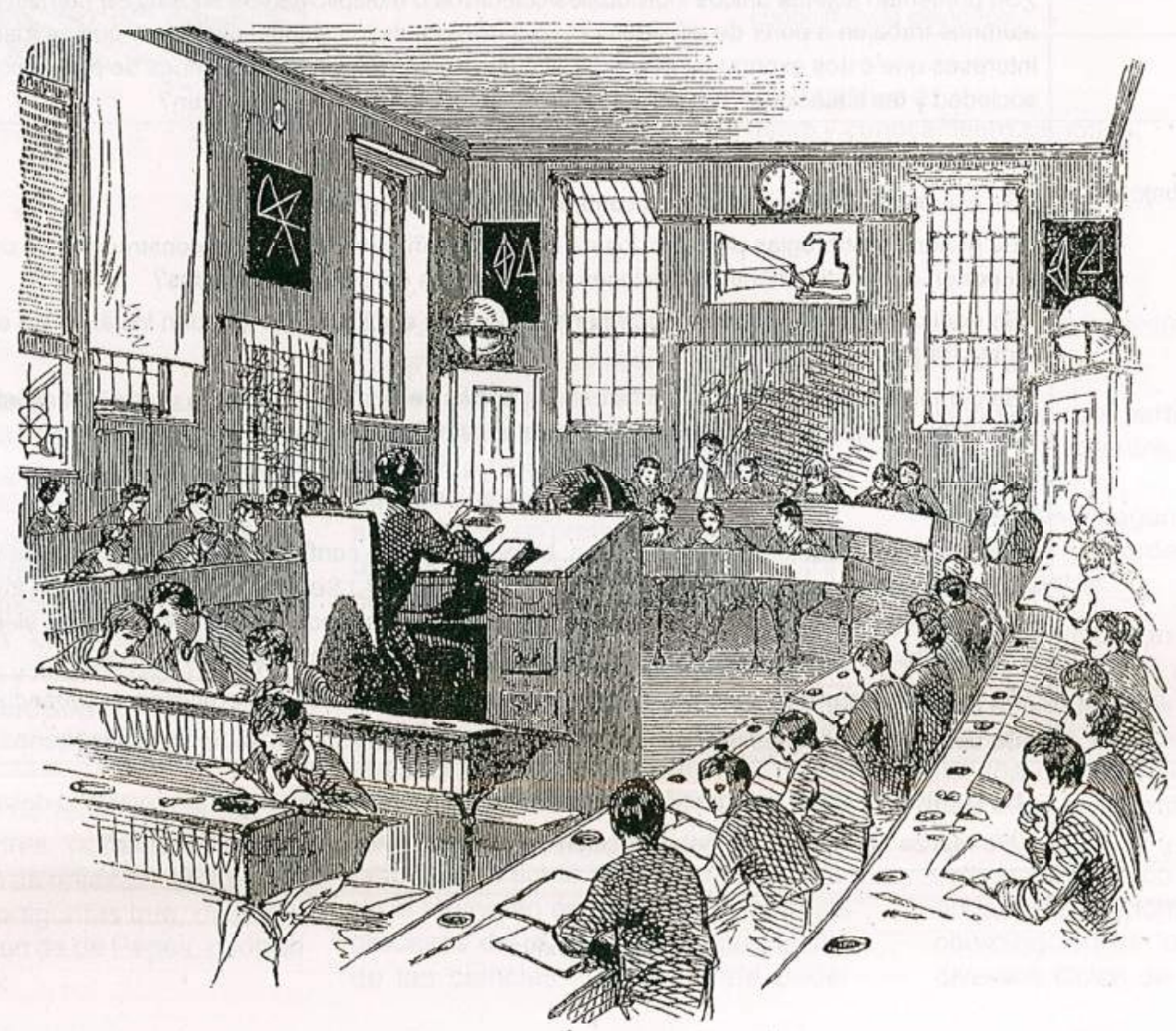

